\title{
Review
}

\section{Insulin regulation of glucose uptake: a complex interplay of intracellular signalling pathways}

\author{
A. H. Khan, J. E. Pessin \\ Department of Physiology and Biophysics, University of Iowa, Iowa City, USA
}

\begin{abstract}
Insulin-stimulated glucose uptake in adipose tissue and striated muscle is critical for reducing post-prandial blood glucose concentrations and the dysregulation of this process is one hallmark of Type II (non-insulin-dependent) diabetes mellitus. It has been well established that the insulin-stimulated redistribution of the insulin responsive glucose transporter, GLUT-4, from intracellular storage sites to the plasma membrane depends on the production of phosphoinositide 3,4,5 trisphosphate by the Class IA Phosphatidylinositol 3' kinase. Recent discoveries however, have shown the presence of a second insulin signalling pathway leading to GLUT-4 translocation, a pathway dependent on insulin receptor signalling emanating from caveolae or lipid rafts at the plasma membrane. This pathway begins with the phosphorylation of the adap-
\end{abstract}

tor protein $\mathrm{Cbl}$ by the insulin receptor, and results in the activation of a small GTP binding protein, TC10, a member of the Rho family. TC10 is able to modulate actin structure in 3T3L1 adipocytes, and its overexpression inhibits insulin-stimulated GLUT-4 translocation, an inhibition completely dependent on localization of TC10 to the caveolae or lipid rafts. The spatial compartmentalization of insulin signalling from caveolae or lipid rafts provides a novel signalling pathway that functions in concert with general signalling mechanisms in the control of actin dynamics regulating insulin-dependent GLUT-4 translocation. [Diabetologia (2002) 45:1475-1483]

Keywords Diabetes, insulin signalling, GLUT-4, actin, TC10, translocation, membrane transport, adipocyte, muscle, PI 3' kinase.
Received: 4 April 2002 / Revised: 12 August 2002

Published online: 18 October 2002

(C) Springer-Verlag 2002

Corresponding author: Dr. J. E. Pessin, The Department of Physiology and Biophysics, The University of Iowa, 51 Newton Road, Iowa City, IA 52242, USA.

E-mail: jeffrey-pessin@uiowa.edu

Abbreviations: IR, Insulin receptor; PTB, protein tyrosine binding; SH2, Src homology 2; PI3K, phosphatidylinositol 3' kinase; PDK-1, 3' phosphoinositide-dependent kinase-1; PKB, protein kinase $\mathrm{B}$; $\mathrm{PKC}$, protein kinase $\mathrm{C}$; $\mathrm{PI}(3,4,5) \mathrm{P} 3$, phosphoinositide 3,4,5 trisphosphate; CAP, Cbl associated protein; APS, associated protein substrate; SH3, Src homology 3; CAAX, cysteine-aliphatic amino acid-any amino acid; WASP, Wiskott-Aldrich syndrome protein; N-WASP, neural WiskottAldrich syndrome protein; Arp, actin related protein; $\mathrm{PI}(4,5)$ P2, phosphoinositide 4,5 bisphosphate; eGFP, enhanced green flourescent protein; GTP $\gamma \mathrm{S}$, guanosine $5^{\prime}$ - $[\gamma$-thio $]$ triphosphate.
Type II (non-insulin-dependent) diabetes mellitus is a clinical disorder of sugar and fat metabolism caused by an inability of insulin to promote sufficient glucose uptake into adipose tissue and striated muscle and to prevent glucose output from the liver. Insulin is the most important hormone in the regulation of blood glucose concentrations and is essential in the postprandial state. As blood sugar concentrations rise, insulin is secreted into the blood stream by the pancreatic beta cell of the endocrine pancreas. Insulin stimulates glucose uptake into fat and muscle to promote the storage of sugar as intracellular triglycerides and glycogen in fat and muscle. In addition, insulin inhibits the production and release of glucose from the liver (gluconeogenesis and glycogenolysis). In a healthy person, this prevents the rise of blood sugar concentrations that would occur after meal ingestion. Howev- 
er, in the early stages of Type II diabetes, blood glucose concentrations remain increased, despite the presence of normal to high insulin concentrations in the bloodstream. The combined inability of muscle and adipose tissue to facilitate glucose uptake and of the liver to suppress glucose output in response to increasing amounts of insulin are referred to as insulin resistance. It is regarded as the hallmark characteristic of Type II diabetes. The later stages of Type II diabetes are characterized by low insulin concentrations and the need for exogenous insulin due to the eventual exhaustion of insulin secretory ability by the pancreatic beta cells. As blood sugar concentrations remain persistently high over many years, clinical complications including retinopathy, nephropathy, neuropathy and cardiovascular disease develop and thereby increase the patient's morbidity and mortality. The high incidence of Type II diabetes and the seriousness of its complications make it imperative to understand the molecular basis of insulin-stimulated glucose transport and insulin resistance. In this review, we survey the established role of the insulin signalling pathway in stimulation of insulin-induced glucose uptake. In addition, we describe a novel insulin signalling pathway that functions through restricted spatial compartmentalization in the plasma membrane that links insulin receptor signalling in lipid microdomains to changes in the actin cytoskeleton.

\section{Glucose transport and the GLUTs}

Glucose transport is mediated through solute carriers referred to as the GLUT family of facilitative glucose transporters, each with different tissue distributions, kinetic properties and sugar specificity. Currently there are 13 members of this family (GLUT-1-12) plus the myo-inositol transporter HMIT1. At present, the best-characterized members of this family are the class I glucose transporters (GLUT-1-4).

The GLUT-1 transporter is ubiquitously expressed and responsible for basal glucose uptake. The GLUT-2 isoform is primarily expressed in beta cells and in the liver, and has a relatively low affinity (high $\mathrm{Km}$ ) for glucose. It serves in combination with hexokinase as part of the glucose sensor. GLUT-3 has the highest affinity (lowest $\mathrm{Km}$ ) and is expressed during fetal development and in adult neurons. Similar to the tightly controlled distribution and functionality of the other GLUT family members, the GLUT- 4 protein is predominantly restricted to fat and muscle and responsible for insulin-stimulated uptake.

The insulin stimulation of glucose uptake in adipose and muscle tissue occurs through a complex and as yet incompletely defined signalling pathway proceeding through the insulin receptor tyrosine kinase. The primary effect is to promote the movement of the GLUT-4 protein from intracellular storage sites to the plasma membrane. In the basal state, GLUT-4 is localized to a morphologically defined "tubulovesicular system" present in the intracellular compartment, while in the presence of insulin, GLUT-4 is immunolocalized to the plasma membrane of fat, skeletal and cardiac muscle $[1,2,3]$. The rate-limiting step at which insulin stimulates uptake of glucose in muscle and fat is the translocation of GLUT-4 transporters to the plasma membrane [4].

\section{The classic pathway of insulin signalling}

The Insulin Receptor is a heterotetrameric membrane protein consisting of two identical $\alpha$ and $\beta$ subunits. Insulin binds to the $\alpha$ subunits of the insulin receptor (IR), thereby activating the intrinsic kinase activity in the $\beta$ subunit. This results in an intramolecular transautophosphorylation reaction whereby one $\beta$ subunit tyrosine phopshorylates the adjacent $\beta$ subunit. The insulin receptor substrate (IRS) family of proteins specifically interacts with the phosphorylated IR through a phosphotyrosine binding (PTB) module, which then facilitates phosphorylation of IRS on a number of tyrosine residues by the activated IR [5]. These phosphotyrosine residues on IRS proteins provide docking sites for proteins with Src Homology 2 (SH2) domains with p85 being the most important regulatory subunit of the Type IA phosphatidylinositol 3' kinase (PI3K). It exists in the cytosol as a dimer of a regulatory p85 subunit and a catalytic p110 subunit [6]. Recruitment of the regulatory subunit brings the catalytic p $110 \alpha$ subunit to the plasma membrane, where it catalyzes the phosphorylation of the 3' position in the inositol ring of phosphoinositide (PI) lipids. Specifically, the Type IA PI3K catalyzes the formation of $\mathrm{PI}(3,4,5)$-trisphosphate from $\mathrm{PI}(4,5)$-bisphosphate, and $\mathrm{PI}(3,4)$-bisphosphate from $\mathrm{PI}(4)$-phosphate [6]. The phosphorylation of the 3 ' position recruits and activates proteins containing pleckstrin homology domains, including the 3' phosphoinositide-dependent kinase-1 (PDK-1) and Akt (also known as protein kinase B) [7]. In turn, PDK-1 phosphorylates and activates downstream effectors including Akt and the atypical protein kinase $\mathrm{C} \zeta / \lambda$ (Fig. 1), The role of this pathway in insulin-stimulated GLUT-4 translocation has been confirmed through a series of studies using chemical and dominant-negative inhibitors of the PI3K. Chemical inhibitors of the PI3K, including wortmannin and LY294002, and biological blockade of the PI3K signalling pathway, including expression of dominant-negative mutants, all inhibit insulin-stimulated GLUT-4 translocation and glucose uptake $[8,9$, $10,11]$. In addition, PI3K blocking antibodies or fusion proteins inhibit GLUT-4 translocation as does degradation of the $\mathrm{PI}(3,4,5) \mathrm{P}_{3}$ by overexpression of $3^{\prime}$ and $5^{\prime}$ specific phosphatases $[10,12,13]$. This evidence suggests that activation of the PI $3 \mathrm{~K}$ and the for- 


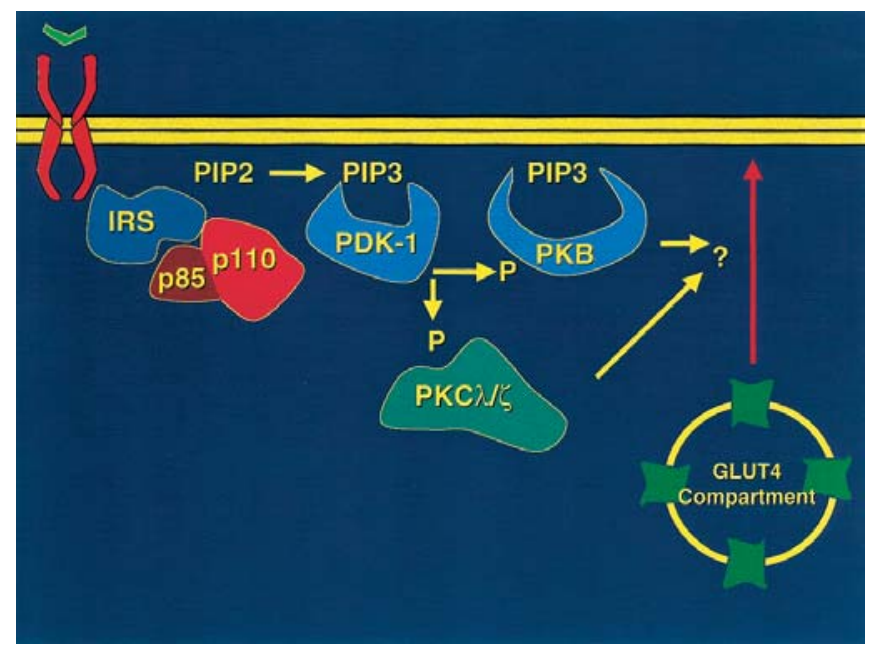

Fig. 1. General insulin signalling pathway leading to GLUT-4 translocation: insulin (green) binds to the insulin receptor (red), activating its tyrosine kinase activity. The activated insulin receptor tyrosine kinase phosphorylates IRS-1 on tyrosine residues allowing for the recruitment of the $\mathrm{p} 85 / \mathrm{p} 110$ phosphatidylinositol 3' kinase (PI3K) to the plasma membrane. Activation and/or recruitment of $\mathrm{PI} 3 \mathrm{~K}$ generates $\mathrm{PI}(3,4,5) \mathrm{P}_{3}$ from $\mathrm{PI}(4,5) \mathrm{P}_{2}$, thereby recruiting the 3 ' phosphoinositide-dependent kinase-1 (PDK-1). PDK-1 phosphorylates and activates both protein kinase $\mathrm{B}$ (PKB/Akt) and the atypical PKC $\lambda / \zeta$ (aPKCs). PKB and the atypical PKCs promote GLUT-4 translocation by an as yet unknown downstream signalling pathway

mation of $\mathrm{PI}(3,4,5) \mathrm{P}_{3}$ is necessary to mediate insulinstimulated GLUT-4 translocation. Furthermore, overexpression of constitutively active forms of the PI3K is able to induce a varying degree of glucose uptake and GLUT-4 translocation in the absence of insulin. Overexpression of a constitutively active form of the catalytic subunit, p110 $\alpha$, increases the basal glucose uptake and GLUT- 4 translocation 50 to $100 \%$ of insulin stimulated concentrations, depending on the study $[14,15,16,17]$. These data confirm that PI3K is necessary for insulin-stimulated GLUT-4 translocation, and could be sufficient to account for the entire insulin-stimulated glucose uptake. However, the role of $\mathrm{PI}(3,4,5) \mathrm{P}_{3}$ and its downstream effectors, mainly Akt and $\mathrm{PKC}$, and their contribution to insulin-stimulated GLUT-4 translocation are controversial.

Clearly Akt is activated in response to insulin stimulation in a variety of cell types [18]. However, the effect of overexpression of dominant-negative Akt mutants on glucose uptake has given mixed results. Whereas expression of the phosphorylation-site-deficient Akt does not affect insulin-stimulated GLUT-4 translocation, a kinase dead and a dual kinase dead and phosphorylation-deficient construct both inhibit GLUT-4 translocation, despite the dominant-negative nature of all three of the constructs $[19,20,21]$. If Akt is necessary for GLUT-4 translocation, then exogenous activation of Akt should stimulate GLUT-4 translocation. Indeed, expression of a constitutively active Akt with a membrane targeting sequence pro- motes GLUT-4 translocation in a hormone-independent manner but this apparently requires a substantially longer time than insulin stimulation $[19,22,23,24$, $25]$. The role of Akt2(PKB $\beta)$, the specific Akt isoform expressed and activated in insulin responsive tissues, in the whole animal shows a complex role for Akt in insulin signalling. Mice deficient in Akt2 are insulin resistant and have higher blood concentrations of glucose and insulin. However, these mice are completely unable to suppress hepatic glucose output (gluconeogenesis) in response to insulin, and have only slightly decreased insulin-stimulated glucose uptake in glycolytic fast-twitch muscle [26], with the former probably accounting for the majority of the insulin resistance. Therefore, the disruption of Akt2 signalling interferes with insulin signalling and glucose homeostasis in both liver and muscle, making it more difficult to assess the importance of Akt signalling in insulin-stimulated GLUT-4 translocation. The role of the other isoforms suggests that they might not play a primary role in insulin signalling and GLUT-4 translocation, as the disruption of Akt1 in mice has no effect on glucose homeostasis. However, the possibility that the Akt1 isoform compensates for the loss of Akt2 in the Akt2 knockout mice remains to be tested [27]. As an alternative to Akt signalling, the atypical PKC isoforms, $\mathrm{PKC}-\lambda$ and $\mathrm{PKC}-\zeta$, are activated by insulin, through a PI3K dependent pathway $[28,29,30]$. Similar to some of the data seen with Akt, overexpression of constitutively active forms of the enzymes increases glucose uptake and GLUT-4 translocation by about 50 to $100 \%$ of that of insulin stimulation [31]. Expression of dominant-negative forms of atypical PKCs containing a mutation in the critical lysine residue in the kinase domain or a mutation on the PDK-1 target phosphorylation site required for activation, inhibits insulin-stimulated glucose uptake and GLUT-4 translocation by about half of the maximal insulin-stimulated uptake [30, 31]. Further evidence for the role of atypical PKCs in insulin stimulated GLUT-4 translocation comes from inhibition by atypical PKC isotype-specific interacting protein (ASIP) overexpression [32].

\section{The PI3K independent pathway of insulin signalling}

Although the necessity of the PI3K pathway is certain, the sufficiency of this pathway alone to promote GLUT-4 translocation and glucose uptake remains unproven. First of all, other growth factors, such as the Platelet-Derived Growth Factor (PDGF), stimulate PI3K activity but are unable to cause GLUT-4 translocation or glucose uptake [33]. Overexpression of an activated form of the PI3K increases GLUT-4 translocation and glucose uptake anywhere from $50 \%$ to $100 \%$ of full insulin-stimulated glucose uptake, yet insulin is able to further increase glucose uptake in the presence of activated $\mathrm{p} 110 \alpha[14,17]$. Further evi- 


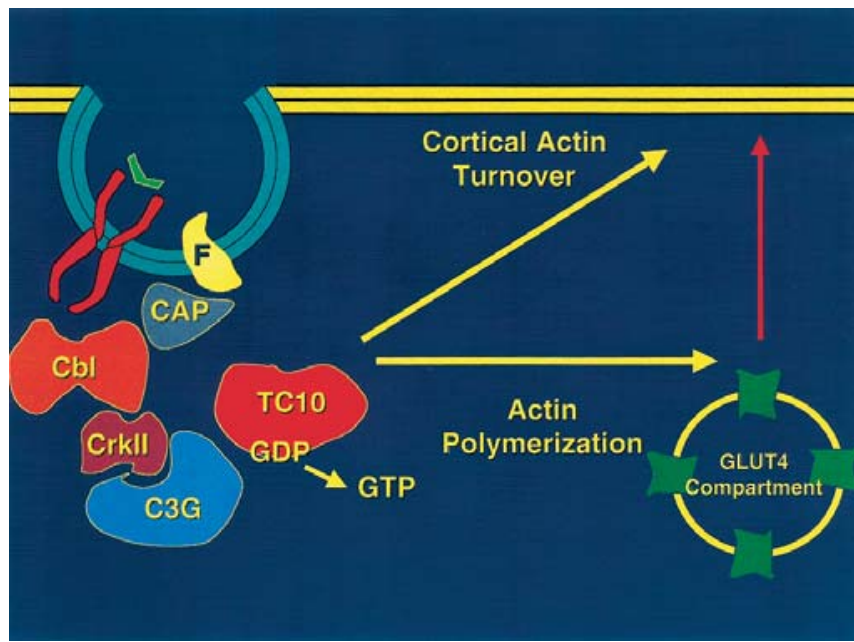

Fig. 2. Hypothesized additional pathway necessary for the insulin stimulation of GLUT-4 translocation: insulin (green) binds to the insulin receptor (red) located in caveolae/lipid rafts in the plasma membrane. Flotillin $(\mathrm{F})$, located in the caveolae/lipid rafts, binds the $\mathrm{Cbl}$ associated protein (CAP). CAP targets $\mathrm{Cbl}$ to the lipid raft domains and the tyrosine phosphorylated $\mathrm{Cbl}$ recruits the $\mathrm{CrkII} / \mathrm{C} 3 \mathrm{G}$ complex. $\mathrm{C} 3 \mathrm{G}$, a guanine nucleotide exchange factor, exchanges GDP for GTP on TC10. Activated, GTP bound TC10 can now participate in GLUT-4 translocation, through modification of cortical actin or stimulating actin polymerization on GLUT-4 compartments

dence to show that activation of the PI3K pathway might not be sufficient for explaining the full insulin stimulated glucose uptake came from studies that used membrane permeant forms of the products of the $\mathrm{PI} 3 \mathrm{~K}$ pathway [34]. $\mathrm{PI}(3,4,5) \mathrm{P}_{3} / \mathrm{AM}$ is an acetoxymethyl derivative that crosses cell membranes into the cytosol where the AM groups are hydrolysed by intracellular esterases, resulting in biologically active $\mathrm{PI}(3,4,5) \mathrm{P}_{3}$. The addition of this $\mathrm{PI}(3,4,5) \mathrm{P}_{3} / \mathrm{AM}$ to 3T3L1 adipocytes had no effect on basal glucose uptake. However, pretreatment with wortmannin, the inhibitor of the PI3K pathway, plus addition of both the $\mathrm{PI}(3,4,5) \mathrm{P}_{3} / \mathrm{AM}$ and insulin resulted in nearly complete restoration of glucose uptake, similar to that seen with insulin alone. This suggests the presence of a second pathway that is also activated by insulin, but is independent of the PI3K pathway and not inhibited by wortmannin. Thus, the above data indicate the presence of an insulin signalling pathway that promotes GLUT-4 translocation independently of the PI3K pathway.

We have proposed a model for a second pathway of insulin signalling required for GLUT-4 translocation and glucose uptake that could proceed through a signalling pathway originating from lipid rafts and resulting in the ultimate activation of a small G-protein, TC10. A central figure in this pathway is the Cbl-associated protein, CAP, and the associated protein substrate, APS [35, 36]. Phosphotyrosine residues on $\mathrm{Cbl}$ provide docking sites for the CrkII/C3G complex [37]. $\mathrm{CrkII}$ is an adaptor protein that links $\mathrm{Cbl}$ to $\mathrm{C} 3 \mathrm{G}$, while $\mathrm{C} 3 \mathrm{G}$ can function as a guanine nucleotide exchange factor for the small GTP binding protein, TC10 [38, 39]. TC10, much like its closest known homologue, $\mathrm{Cdc} 42$, has profound effects on actin structure, and is thought to contribute to the maintenance of actin structure in 3T3L1 adipocytes. Transcripts of both CAP and TC10 are enriched in skeletal and cardiac muscle, further suggesting a specific role in insulin signalling to glucose uptake $[35,40]$. Therefore, we propose that this PI3K independent pathway appears to link actin dynamics to GLUT-4 translocation (Fig. 2).

Although APS provides an important adaptor function required for tyrosine phosphorylation of Cbl by the insulin receptor [41], the Cbl-associated protein, CAP, seems to play a critical role in the membrane targeting of tyrosine phosphorylated $\mathrm{Cbl}$ [38]. CAP contains an amino terminal Sorbin homology domain, and 3 carboxyl terminal Src homology 3 (SH3) domains. The sorbin homology domain binds flotillin, a component of lipid rafts $[42,43]$, while the third SH3 domain (from the amino terminus) is responsible for the binding interaction between $\mathrm{Cbl}$ and CAP. Thus, these data suggest that CAP plays the role of an adaptor linking $\mathrm{Cbl}$ to the plasma membrane lipid raft microdomains through the interaction of CAP with flotillin. Consistent with this interpretation, overexpression of a $\mathrm{Cbl}$ binding deficient $\mathrm{CAP} \triangle \mathrm{SH} 3$ mutant, but not full-length CAP, inhibits GLUT-4 translocation and glucose uptake independently of the activation of the PI3K or MAPK pathways [38]. Lipid raft microdomains contain a unique set of membrane lipids, including sphingolipids and cholesterol, form their own liquid ordered phase in the plasma membrane and do not laterally mix with the other lipids, including phospholipids [44]. Lipid rafts share a unique protein constituency relative to the non-lipid raft plasma membrane, including GPI-anchored proteins, caveolin, flotillin and palmitoylated proteins. Caveolae are a specific kind of lipid raft found in the greatest abundance in adipocytes, skeletal muscle and endothelial cells. Caveolae ("little caves") are 50 to $80 \mathrm{~nm}$ invaginations of the plasma membrane that contain the protein caveolin. In 3T3L1 adipocytes, multiple caveolae are viewed by confocal immunofluorescence in the plasma membrane as a multi-ordered structure that forms a donut-like complex about $1 \mathrm{mi}-$ cron in diameter that can be resolved using confocal microscopy [45, 46]. Electron micrographs show that the insulin receptor in the plasma membrane is present in caveolae [45]. However, the effect of disruption of caveolae with the addition of cholesterol extracting agents, like $\beta$-cyclodextrin or filipin has yielded mixed results. In the case of $\beta$-cyclodextrin, treatment reduces insulin receptor activity, downstream insulin signalling, including IRS-1 and Akt phosphorylation, and insulin-stimulated glucose uptake in a dose dependent manner $[45,47]$. However, nystatin and fili- 
pin, both cholesterol chelating compounds, have no effect on insulin-stimulated glucose uptake [48]. Cholesterol sequestration through the expression of the dominant negative Cav3/DGV in 3T3L1 adipocytes inhibits insulin-stimulated GLUT-4 translocation while the full-length caveolin 3 has no effect [46]. Thus, lipid raft microdomains are thought to provide a necessary structural and/or functional component for insulin-stimulated glucose uptake in 3T3L1 adipocytes.

The recruitment and phosphorylation of $\mathrm{Cbl}$ to lipid raft microdomains in response to insulin permits the recruitment of the adaptor protein, CrkII. CrkII is an adaptor protein containing an $\mathrm{SH} 2$ domain that binds to tyrosine phosphorylated $\mathrm{Cbl}$, and $\mathrm{Cb} 2$ tandem $\mathrm{SH} 3$ domains with the central $\mathrm{SH} 3$ domain responsible for the binding to $\mathrm{C} 3 \mathrm{G}$ [49]. The CrkII SH3 domain binds to a proline-rich motif in $\mathrm{C} 3 \mathrm{G}$ in a constitutive manner and seems to provide a mechanism for localizing the $\mathrm{C} 3 \mathrm{G}$ protein. By virtue of its binding to phosphorylated $\mathrm{Cbl}$, the CrkII-C3G complex is therefore recruited to the lipid raft microdomains of 3T3L1 adipocytes in response to insulin stimulation. This recruitment is blocked by overexpression of the CAP $\Delta \mathrm{SH} 3$ mutant. These data indicate that these proteins lie in the same continuous signalling pathway [39].

$\mathrm{C} 3 \mathrm{G}$ is a known guanine nucleotide exchange factor for the small G-protein Rap1 [50]. Additionally, in vitro, C3G catalyzes nucleotide exchange on TC10, a small G-protein, while Sos, a known exchange factor for Ras, was unable to do the same. TC10, a small Gprotein of the Rho family, is activated in vivo with insulin stimulation, as measured by its binding to a P21activated kinase (PAK) binding domain. This activation is blocked by the co-expression of the CAP $\Delta \mathrm{SH} 3$ mutant [39]. However, activation of TC10 occurs even in the presence of wortmannin, providing more evidence that TC10 and its upstream signalling components are involved in an insulin signalling pathway that is independent of the PI3K pathway. Like the functional effect of CAP $\Delta \mathrm{SH} 3$, overexpression of either wild-type or a dominant negative TC10, but not its closest known homologue, Cdc42 inhibits insulinstimulated GLUT-4 translocation in 3T3L1 adipocytes. This provides further proof for a functionally important role for a pathway linking insulin signalling in caveolae to insulin-stimulated GLUT-4 translocation.

\section{TC10 and lipid raft localization}

TC10 is a close homologue of $\mathrm{Cdc} 42$, with a sequence identity of $69 \%$ and sequence similarity of $83 \%$. Therefore the obvious question arises: why overexpression of TC10, but not Cdc42, inhibits insulinstimulated GLUT-4 translocation. Given the recruitment of $\mathrm{Cbl}$, and therefore the CrkII/C3G complex, to caveolae, proper membrane localization of the small G-protein could distinguish its ability to participate in or disrupt the signalling pathway. The membrane localization of small G-proteins is determined by posttranslational modifications in their carboxyl terminal tail. In this regard, $\mathrm{TC} 10$ and $\mathrm{Cdc} 42$ display a distinct divergence at their carboxyl terminal CAAX sequences that undergoes post-translational modification at the carboxyl terminal cysteine residues. Cdc42 contains one carboxyl terminal residue that is subjected to geranylgeranylation whereas the TC10 CAAX box provides a sequence context for farnesylation. More importantly, TC10 but not Cdc42 contains two additional upstream cysteine residues that can undergo palmitoylation. The presence of these additional palmitoylation residues in TC10 is similar to that observed for $\mathrm{H}-\mathrm{Ras}$ which are required for its localization to lipid raft microdomains [51, 52]. In contrast, $\mathrm{K}$-Ras contains a single cysteine that undergoes farnesylation but instead of any further cysteine modification contains a putative phospholipid binding polybasic stretch upstream of the CAAX motif that targets $\mathrm{K}$-Ras to non-lipid raft domains in the plasma membrane.

In 3T3L1 adipocytes, TC10 localizes to the caveolar donut structures. Given that the targeting of H-ras to lipid rafts and K-ras to the non-lipid raft plasma membrane is well established, chimeras of TC10 with the carboxy terminal 19 amino acids replaced with the homologous region of either H-Ras or K-Ras provide an opportunity to investigate the importance of the Cterminus for localization and to determine whether localization defines functionality. A TC10/H-Ras chimera targets to caveolin-1 containing rosette structures in the plasma membrane, and by virtue of this localization inhibits insulin-stimulated GLUT-4 translocation. In contrast, the TC10/K-Ras chimera, which should be targeted to non-lipid raft plasma membrane, is localized in a more diffuse pattern in the plasma membrane and does not have an inhibitory effect on insulin-stimulated GLUT-4 translocation. As with the inhibitory effect of TC10 on GLUT-4 translocation, insulin-stimulated activation of TC10 only occurs when TC10 is targeted to lipid rafts via the wild-type or H-Ras but not the K-Ras carboxy terminus, further suggesting that the upstream signal that activates TC10 is present only in lipid rafts [46].

Given the importance of TC10 localization to function, it brings up the question of the downstream effects and effectors of TC10. Both TC10 and Cdc42 are members of the Rho family of small GTPases; they have nearly identical effector binding regions and therefore might share effectors [40]. The Rho family of GTPases is prominently involved in regulating the actin cytoskeleton, with overexpression of constitutively active Rac in fibroblasts creating lamellipodia and membrane ruffling, Rho expression inducing actin stress fibre remodelling, and $\mathrm{Cdc} 42$ expression pro- 
moting outgrowth of microspikes and filopodia [53]. In fibroblasts, overexpression of a constitutively active, GTPase deficient TC10 resulted in prominent microspike formation, illustrating that TC10 can affect actin dynamics in cells [40]. Therefore, a possible avenue of TC10 action on insulin-stimulated GLUT-4 translocation is to regulate the actin cytoskeleton.

\section{Actin and GLUT-4 translocation}

Since the GLUT-4 glucose transporter travels from intracellular compartments to the plasma membrane, an obvious level of regulation is the cytoskeleton, in particular the actin cytoskeleton. The application of actin modifying drugs like cytochalasin D which is an actin filament capping protein that inhibits actin filament assembly and latrunculin B which is an actin monomer sequestering molecule, both inhibit insulin-stimulated glucose uptake and GLUT-4 translocation in isolated rat and 3T3L1 adipocytes [54, 55, 56]. However, the activation of TC10 by insulin has given new impetus to study the actin cytoskeleton in the context of a signalling pathway activated in response to insulin and involved in promoting GLUT-4 translocation.

Adipocytes have a unique actin cytoskeleton. Unlike the long stress fibres present in fibroblasts, adipocytes have a layer of short actin filaments underneath the plasma membrane, known as cortical actin [56]. C. dificile toxin $\mathrm{B}$, an inactivator of Rho GTPases, inhibits insulin-stimulated GLUT-4 translocation and glucose uptake, presumably through the inactivation of TC10, in concordance with overexpression studies. Similarly, Toxin B and overexpression of TC10 also disrupt the cortical actin present underneath the plasma membrane, suggesting that the mere presence of the actin cytoskeleton could be necessary for insulininduced GLUT-4 translocation. However, the role of actin might not be so simple as the incubation of 3T3L1 adipocytes with jasplakinolide, an actin-filament-binding protein that stabilizes actin filaments, also inhibits insulin-induced GLUT-4 translocation and glucose uptake, despite the presence of actin filaments beneath the plasma membrane. Therefore, the presence or absence of actin filaments might not be as important to GLUT-4 translocation as an insulin-induced turnover of actin filaments near the plasma membrane.

A wealth of knowledge has been uncovered about the role of the downstream effectors of the Rho family of GTPases, most prominently Cdc42, in modifying actin structure in cells. Activated $\mathrm{Cdc} 42$ binds and activates a number of proteins, and amongst the best characterized of these in modifying actin structure is the Wiskott-Aldrich Syndrome Protein (WASP) [53]. Patients with a mutation in WASP have defects in platelet morphology, macrophage chemotaxis and $\mathrm{T}$ cell and B-cell function resulting in a number of clini- cal complications including thrombocytopenia, eczema and immunodeficiency. WASP and its ubiquitous homologue, N-WASP are multi-domain proteins that bind to activated Rho family members, including Cdc42, and Phosphatidylinositol $(4,5)$ bisphosphate $\left(\mathrm{PI}(4,5) \mathrm{P}_{2}\right)$ which activates WASP/N-WASP to bind to the Actin Related Protein 2/3 (Arp2/3) complex of proteins $[57,58]$. The Arp $2 / 3$ complex promotes actin filament polymerization from pre-existing actin filaments by capping the "pointed" (slow growing) end of actin filaments thereby stabilizing the "barbed" (fast growing) end, binding to the side of filaments and forming branches to cross link filaments, and by weakly nucleating filaments on its own [59]. For example, the Arp $2 / 3$ complex is recruited to the leading edge of lamellipodia in hormone activated fibroblasts and pseudopodia at the leading edge of neutrophils stimulated with chemoattractants $[57,59,60]$. Activated small G-proteins recruit the Arp2/3 complex via N-WASP to sites of activated signalling complexes, and thereby link signalling to actin dynamics.

Of interest, the Arp $2 / 3$ complex has also been implicated in the intracellular spread of pathogens including Listeria monocytogenes, Shigella flexneri, and vaccinia virus [61, 62]. The Listeria protein ActA binds the Arp2/3 complex and co-opts its actin polymerization ability to propel the bacterium into neighbouring cells. The bacteria moves through the cytoplasm of an infected cell with a large bundle of actin treadmilling, referred to as an "actin comet tail". Similarly, the Shigella protein, IcsA, binds and activates N-WASP to achieve a similar effect, and the Vaccinia coat protein, A36R, also activates N-WASP through the adapter protein Nck to promote intracellular movement of the viral particles. While this shows the case of a parasitic organism subverting cellular machinery for its own purpose, there is some evidence for actin "comet tailing" in a non-pathogenic setting. $\mathrm{Cdc} 42$ induces actin polymerization on small vesicles in vitro in Xenopus egg extracts that contain only GTP $\gamma \mathrm{S}$ and Rhodamine actin in an N-WASP dependent manner [6364, 65], in neutrophil extracts [66, 67], or on purified PI(4,5)P2 vesicles [68]. In a cellular setting actin accumulates in a similar manner around pinocytic vesicles in mast cells, upon raft derived vesicles in cells overexpressing a phosphatidylinositol phosphate 5' kinase, and in lysosomes of $\mathrm{Xe}$ nopus oocytes treated with diacylglycerol to activate PKC [6970, 71]. While there is not enough data to convincingly say that actin comet tailing occurs physiologically in vivo, one can predict and test whether TC10 have a role in propelling GLUT-4 vesicles via an N-WASP dependent mechanism.

In an in vitro setting containing only Xenopus extracts and a high speed membrane fraction containing GLUT-4-eGFP vesicles, adding GTP $\gamma \mathrm{S}$ or sodium vanadate or insulin pretreatment can promote actin comet tailing on GLUT-4-eGFP vesicles [72]. These 
comet tails are inhibited by dominant-negative $\mathrm{N}$ WASP or by latrunculin B treatment, suggesting an NWASP dependent actin polymerization. In addition, insulin stimulates rhodamine actin comet tailing on GLUT-4-EGFP vesicles in broken, gently sheared adipocytes, giving rise to the possibility that insulin stimulates comet tailing in vivo as a driving force for GLUT-4 translocation. However, overexpression of an $\mathrm{N}$-WASP dominant negative construct that lacks the ability to link to Arp2/3 (N-WASP $\Delta$ VCA) has only a $30 \%$ inhibitory effect on overall insulin-stimulated GLUT-4-EGFP translocation, in comparison to the expression of wild-type N-WASP which has no effect [72]. Intriguingly, overexpression of N-WASP $\triangle$ VCA does not inhibit cortical actin in 3T3L1 adipocytes, suggesting that N-WASP might not be the TC10 effector responsible for cortical actin remodelling. Therefore, while N-WASP could be involved in moving GLUT-4 vesicles within the cell, its overall role in insulin-stimulated GLUT-4 translocation might not be as great. However, this does suggest that TC10 could have dual effects of stimulating actin comet tailing on GLUT-4 vesicles and maintenance of the cortical actin required from GLUT-4 translocation, probably through the use of separate effector proteins.

\section{Conclusions}

A commonality present in both of the insulin signalling pathways is how much remains unknown regarding the molecular mechanisms for insulin-stimulated GLUT-4 translocation. In the classic pathway, the precise roles of Akt PKC $\lambda / \zeta$ in propagating a further downstream signal remain enigmatic. For example, what are their substrates, do they work together or are they redundant, and how do they directly contribute to insulin-stimulated GLUT-4 translocation and glucose uptake? The PI3K independent pathway is still a work-in-progress, but provides a logical framework in which to test hypotheses and ideas. Clearly TC10 is a focal point and it is important to determine through which effectors does TC10 modify cortical actin structure, what is the functional role of TC10 in actin comet tailing, and ultimately, how does actin and other cytoskeletal elements work to promote GLUT-4 translocation? The understanding of these basic processes of insulin action on cells, tissues and humans will ultimately aid in the understanding of insulin resistance and Type II diabetes, and will help to clarify how to therapeutically treat a disease as complex as diabetes.

\section{References}

1. Slot JW, Geuze HJ, Gigengack S, Lienhard GE, James DE (1991) Immuno-localization of the insulin regulatable glucose transporter in brown adipose tissue of the rat. J Cell Biol 113: 123-135
2. Slot JW, Geuze HJ, Gigengack S, James DE, Lienhard GE (1991) Translocation of the glucose transporter GLUT4 in cardiac myocytes of the rat. Proc Natl Acad Sci USA 88: 7815-7819

3. Smith RM, Charron MJ, Shah N, Lodish HF, Jarett L (1991) Immunoelectron microscopic demonstration of insulin-stimulated translocation of glucose transporters to the plasma membrane of isolated rat adipocytes and masking of the carboxyl-terminal epitope of intracellular GLUT4. Proc Natl Acad Sci USA 88: 6893-6897

4. Saltiel AR (2001) New perspectives into the molecular pathogenesis and treatment of type 2 diabetes. Cell 104: 517-529

5. Sesti G, Federici M, Hribal ML et al. (2001) Defects of the insulin receptor substrate (IRS) system in human metabolic disorders. FASEB J 15: 2099-2111

6. Fruman DA, Meyers RE, Cantley LC (1998) Phosphoinositide kinases. Annu Rev Biochem 67: 481-507

7. Rameh LE, Cantley LC (1999) The role of phosphoinositide 3-kinase lipid products in cell function. J Biol Chem 274: 8347-8350

8. Cheatham B, Vlahos CJ, Cheatham L et al. (1994) Phosphatidylinositol 3-kinase activation is required for insulin stimulation of pp70 S6 kinase, DNA synthesis, and glucose transporter translocation. Mol Cell Biol 14: 4902-4911

9. Okada T, Kawano Y, Sakakibara R, Hazeki O, Ui M (1994) Essential role of phophatidylinositol 3-kinase in insulin-induced glucose transport and antilypolysis in rat adipocytes. Studies with a selective inhibitor wortmannin. J Biol Chem 269: 3568-3573

10. Haruta T, Morris AJ, Rose DW et al. (1995) Insulin-stimulated GLUT4 translocation is mediated by a divergent intracellular signaling pathway. J Biol Chem 270: 2799127994

11. Kotani K, Carozzi AJ, Sakaue H et al. (1995) Requirement for phosphoinositide 3-kinase in insulin-stimulated GLUT4 translocation in 3T3-L1 adipocytes. Biochem Biophys Res Commun 209: 343-348

12. Ono H, Katagiri H, Funaki M et al. (2001) Regulation of phosphoinositide metabolism, Akt phosphorylation, and glucose transport by PTEN (phosphatase and tensin homolog deleted on chromosome 10) in 3T3-L1 adipocytes. Mol Endocrinol 15: 1411-1422

13. Vollenweider P, Clodi M, Martin SS et al. (1999) An SH2 domain-containing 5' inositolphosphatase inhibits insulininduced GLUT4 translocation and growth factor-induced actin filament rearrangement. Mol Cell Biol 19: 1081-1091

14. Katagiri H, Asano T, Ishihara H et al. (1996) Overexpression of catalytic subunit $\mathrm{p} 110 \alpha$ of phosphatidylinositol 3kinase increases glucose transport activity with translocation of glucose transporters in 3T3-L1 adipocytes. J Biol Chem 271: 16987-16990

15. Martin SS, Haruta T, Morris AJ et al. (1996) Activated phosphatidylinositol 3-kinase is sufficient to mediate actin rearrangement and GLUT4 translocation in 3T3-L1 adipocytes. J Biol Chem 271: 17605-17608

16. Tanti J-F, Gremeaux T, Grillo S et al. (1996) Overexpression of a constitutively active form of phosphatidylinositol 3-kinase is sufficient to promote Glut4 translocation in adipocytes. J Biol Chem 271: 25227-25232

17. Frevert EU, Kahn BB (1997) Differential effects of constitutively active phosphatidylinositol 3-kinase on glucose transport, glycogen synthase activity, and DNA synthesis in 3T3-L1 adipocytes. Mol Cell Biol 17: 190-198

18. Kohn AD, Kovacina KS, Roth RA (1995) Insulin stimulates the kinase activity of RAC-PK, a pleckstrin homology domain containing ser/thr kinase. EMBO J 14: 4288-4295 
19. Cong LN, Chen H, Li Y et al. (1997) Physiological role of Akt in insulin-stimulated translocation of GLUT4 in transfected rat adipose cells. Mol Endocrinol 11: 1881-1890

20. Kitamura T, Ogawa W, Sakaue H et al. (1998) Requirement for activation of the serine-threonine kinase Akt (protein kinase B) in insulin stimulation of protein synthesis but not of glucose transport. Mol Cell Biol 18: 3708-3717

21. Wang Q, Somwar R, Bilan PJ et al. (1999) Protein kinase B/Akt participates in GLUT4 translocation by insulin in L6 myoblasts. Mol Cell Biol 19: 4008-4018

22. Kohn AD, Summers SA, Birnbaum MJ, Roth RA (1996) Expression of a constitutively active Akt ser/thr kinase in 3T3-L1 adipocytes stimulates glucose uptake and glucose transporter 4 translocation. J Biol Chem 271: 31372-31378

23. Tanti JF, Grillo S, Gremeaux T et al. (1997) Potential role of protein kinase B in glucose transporter 4 translocation in adipocytes. Endocrinology 138: 2005-2010

24. Kohn AD, Barthel A, Kovacina KS et al. (1998) Construction and characterization of a conditionally active version of the serine/threonine kinase Akt. J Biol Chem 273: 11937-11943

25. Foran PG, Fletcher LM, Oatey PB et al. (1999) Protein kinase B stimulates the translocation of GLUT4 but not GLUT1 or transferrin receptors in 3T3-L1 adipocytes by a pathway involving SNAP-23, synaptobrevin-2, and/or cellubrevin. J Biol Chem 274: 28087-28095

26. Cho H, Mu J, Kim JK et al. (2001) Insulin resistance and a diabetes mellitus-like syndrome in mice lacking the protein kinase Akt2 (PKB beta). Science 292: 1728-1731

27. Cho H, Thorvaldsen JL, Chu Q, Feng F, Birnbaum MJ (2001) Akt1/pkbalpha is required for normal growth but dispensable for maintenance of glucose homeostasis in mice. J Biol Chem 276: 38349-38352

28. Bandyopadhyay G, Standaert ML, Zhao L et al. (1997) Activation of protein kinase $\mathrm{C}$ (alpha, beta, and zeta) by insulin in 3T3/L1 cells. Transfection studies suggest a role for PKC-zeta in glucose transport. J Biol Chem 272: 25512558

29. Standaert ML, Galloway L, Karnam P et al. (1997) Protein kinase C-zeta as a downstream effector of phosphatidylinositol 3-kinase during insulin stimulation in rat adipocytes. Potential role in glucose transport. J Biol Chem 272: 30075-30082

30. Kotani K, Ogawa W, Matsumoto M et al. (1998) Requirement of Atypical Protein Kinase Clambda for Insulin Stimulation of Glucose Uptake but Not for Akt Activation in 3T3-L1 Adipocytes. Mol Cell Biol 18: 6971-6982

31. Bandyopadhyay G, Standaert ML, Sajan MP et al. (1999) Dependence of insulin-stimulated glucose transporter 4 translocation on 3-phosphoinositide-dependent protein kinase- 1 and its target threonine-410 in the activation loop of protein kinase C-zeta. Mol Endocrinol 13: 1766-1772

32. Kotani K, Ogawa W, Hashiramoto M et al. (2000) Inhibition of insulin-induced glucose uptake by atypical protein kinase $\mathrm{C}$ isotype-specific interacting protein in 3T3-L1 adipocytes. J Biol Chem 275: 26390-26395

33. Summers SA, Whiteman EL, Cho H, Lipfert L, Birnbaum MJ (1999) Differentiation-dependent suppression of platelet-derived growth factor signaling in cultured adipocytes. J Biol Chem 274: 23858-23867

34. Jiang T, Sweeney G, Rudolf MT et al. (1998) Membranepermeant esters of phosphatidylinositol 3,4,5-trisphosphate. J Biol Chem 273: 11017-11024

35. Ribon V, Printen JA, Hoffman NG, Kay BK, Saltiel AR (1998) A novel, multifuntional c-Cbl binding protein in insulin receptor signaling in 3T3-L1 adipocytes. Mol Cell Biol 18: 872-879
36. Moodie SA, Alleman-Sposeto J, Gustafson TA (1999) Identification of the APS protein as a novel insulin receptor substrate. J Biol Chem 274: 11186-11193

37. Tanaka S, Morishita T, Hashimoto Y et al. (1994) C3G, a Guanine Nucleotide-Releasing Protein Expressed Ubiquitously, Binds to the SRC Homology-3 Domains of CRK and GRB2/ASH Proteins. Proc Natl Acad Sci USA 91: 3443-3447

38. Baumann CA, Ribon V, Kanzaki M et al. (2000) CAP defines a second signalling pathway required for insulin-stimulated glucose transport. Nature 407: 202-207

39. Chiang SH, Baumann CA, Kanzaki M et al. (2001) Insulinstimulated GLUT4 translocation requires the CAP-dependent activation of TC10. Nature 410: 944-948

40. Neudauer CL, Joberty G, Tatsis N, Macara IG (1998) Distinct cellular effects and interactions of the Rho-family GTPase TC10. Curr Biol 8: 1151-1160

41. Ahmed Z, Smith BJ, Pillay TS (2000) The APS adapter protein couples the insulin receptor to the phosphorylation of $\mathrm{c}-\mathrm{Cbl}$ and facilitates ligand-stimulated ubiquitination of the insulin receptor. FEBS Lett 475: 31-34

42. Bickel PE, Scherer PE, Schnitzer JE et al. (1997) Flotillin and epidermal surface antigen define a new family of caveolae-associated integral membrane proteins. J Biol Chem 272: 13793-13802

43. Kimura A, Baumann CA, Chiang SH, Saltiel AR (2001) The sorbin homology domain: a motif for the targeting of proteins to lipid rafts. Proc Natl Acad Sci USA 98: 9098-9103

44. Smart EJ, Graf GA, McNiven MA et al. (1999) Caveolins, liquid-ordered domains, and signal transduction. Mol Cell Biol 19: 7289-7304

45. Gustavsson J, Parpal S, Karlsson M et al. (1999) Localization of the insulin receptor in caveolae of adipocyte plasma membrane. FASEB J 13: 1961-1971

46. Watson RT, Shigematsu S, Chiang SH et al. (2001) Lipid raft microdomain compartmentalization of $\mathrm{TC} 10$ is required for insulin signaling and GLUT4 translocation. J Cell Biol 154: 829-840

47. Parpal S, Karlsson M, Thorn H, Stralfors P (2001) Cholesterol depletion disrupts caveolae and insulin receptor signaling for metabolic control via insulin receptor substrate1 , but not for mitogen-activated protein kinase control. J Biol Chem 276: 9670-9678

48. Ros-Baro A, Lopez-Iglesias C, Peiro S et al. (2001) Lipid rafts are required for GLUT4 internalization in adipose cells. Proc Natl Acad Sci USA 98: 12050-12055

49. Matsuda M, Tanaka S, Nagata S et al. (1992) Two species of human CRK cDNA encode proteins with distinct biological activities. Mol Cell Biol 12: 3482-3489

50. Gotoh T, Hattori S, Nakamura S et al. (1995) Identification of Rap1 as a target for the Crk SH3 domain-binding guanine nucleotide-releasing factor $\mathrm{C} 3 \mathrm{G}$. Mol Cell Biol 15: 6746-6753

51. Roy S, Luetterforst R, Harding A et al. (1999) Dominantnegative caveolin inhibits H-Ras function by disrupting cholesterol-rich plasma membrane domains. Nat Cell Biol 1: $98-105$

52. Apolloni A, Prior IA, Lindsay M, Parton RG, Hancock JF (2000) H-ras but not K-ras traffics to the plasma membrane through the exocytic pathway. Mol Cell Biol 20: 2475-2487

53. Bishop AL, Hall A (2000) Rho GTPases and their effector proteins. Biochem J 348: 241-255

54. Tsakiridis T, Vranic M, Klip A (1994) Disassembly of the actin network inhibits insulin-dependent stimulation of glucose transport and prevents recruitment of glucose transporters to the plasma membrane. J Biol Chem 269: 29934-29942 
55. Wang Q, Bilan PJ, Tsakiridis T, Hinek A, Klip A (1998) Actin filaments participate in the relocalization of phosphatidylinositol3-kinase to glucose transporter-containing compartments and in the stimulation of glucose uptake in 3T3-L1 adipocytes. Biochem J 331: 917-928

56. Kanzaki M, Pessin JE (2001) Insulin-stimulated GLUT4 Translocation in Adipocytes Is Dependent upon Cortical Actin Remodeling. J Biol Chem 276: 42436-42444

57. Machesky LM, Insall RH (1999) Signaling to actin dynamics. J Cell Biol 146: 267-272

58. Zigmond SH (2000) How WASP regulates actin polymerization. J Cell Biol 150: 117-120

59. Welch MD (1999) The world according to Arp: regulation of actin nucleation by the Arp2/3 complex. Trends Cell Biol 9: 423-427

60. Weiner OD, Servant G, Welch MD et al. (1999) Spatial control of actin polymerization during neutrophil chemotaxis. Nat Cell Biol 1: 75-81

61. Frischknecht F, Moreau V, Rottger S et al. (1999) Actinbased motility of vaccinia virus mimics receptor tyrosine kinase signalling. Nature 401: 926-929

62. Egile C, Loisel TP, Laurent V et al. (1999) Activation of the CDC42 effector N-WASP by the Shigella flexneri IcsA protein promotes actin nucleation by Arp $2 / 3$ complex and bacterial actin-based motility. J Cell Biol 146: 1319-1332

63. Ma L, Cantley LC, Janmey PA, Kirschner MW (1998) Corequirement of specific phosphoinositides and small GTPbinding protein $\mathrm{Cdc} 42$ in inducing actin assembly in Xenopus egg extracts. J Cell Biol 140: 1125-1136

64. Ma L, Rohatgi R, Kirschner MW (1998) The Arp2/3 complex mediates actin polymerization induced by the small GTP-binding protein Cdc42. Proc Natl Acad Sci USA 95: 15362-15367
65. Rohatgi R, Ma L, Miki H et al. (1999) The interaction between N-WASP and the Arp2/3 complex links Cdc42-dependent signals to actin assembly. Cell 97: 221-231

66. Zigmond SH, Joyce M, Borleis J, Bokoch GM Devreotes PN (1997) Regulation of actin polymerization in cellfree systems by GTPgammaS and Cdc42. J Cell Biol 138: 363-374

67. Zigmond SH, Joyce M, Yang C et al. (1998) Mechanism of Cdc42-induced actin polymerization in neutrophil extracts. J Cell Biol 142: 1001-1012

68. Higgs HN, Pollard TD (2000) Activation by Cdc42 and PIP(2) of Wiskott-Aldrich syndrome protein (WASp) stimulates actin nucleation by Arp2/3 complex. J Cell Biol 150: 1311-1320

69. Merrifield CJ, Moss SE, Ballestrem C et al. (1999) Endocytic vesicles move at the tips of actin tails in cultured mast cells. Nat Cell Biol 1: 72-74

70. Rozelle AL, Machesky LM, Yamamoto M et al. (2000) Phosphatidylinositol 4,5-bisphosphate induces actin-based movement of raft-enriched vesicles through WASP-Arp2/3. Curr Biol 10: 311-320

71. Taunton J, Rowning BA, Coughlin ML et al. (2000) Actindependent propulsion of endosomes and lysosomes by recruitment of N-WASP. J Cell Biol 148: 519-530

72. Kanzaki M, Watson RT, Khan AH, Pessin JE (2001) Insulin stimulates actin comet tails on intracellular GLUT4containing compartments in differentiated 3T3L1 adipocytes. J Biol Chem 276: 49331-49336 